\title{
Coriander (Coriandrum sativum L.) : a potential source of high-value components for functional foods and nutraceuticals - a review
}

\begin{abstract}
Coriander (Coriandrum sativum L.), a herbal plant, belonging to the family Apiceae, is valued for its culinary and medicinal uses. All parts of this herb are in use as flavoring agent and/or as traditional remedies for the treatment of different disorders in the folk medicine systems of different civilizations. The plant is a potential source of lipids (rich in petroselinic acid) and an essential oil (high in linalool) isolated from the seeds and the aerial parts. Due to the presence of a multitude of bioactives, a wide array of pharmacological activities have been ascribed to different parts of this herb, which include anti-microbial, anti-oxidant, antidiabetic, anxiolytic, anti-epileptic, anti-depressant, anti-mutagenic, anti-inflammatory, antidyslipidemic, anti-hypertensive, neuro-protective and diuretic. Interestingly, coriander also possessed lead-detoxifying potential. This review focuses on the medicinal uses, detailed phytochemistry, and the biological activities of this valuable herb to explore its potential uses as a functional food for the nutraceutical industry.
\end{abstract}

Keyword: Coriander; Medicinal uses; Functional food; Essential oil; Lipids; Linalool; Biological activities 